\title{
Effects of Transcranial Static Magnetic Stimulation on Motor Cortex Evaluated by Different TMS Waveforms and Current Directions
}

\author{
Paula Davila-Pérez ${ }^{\mathrm{a}, \mathrm{b}}$, Alvaro Pascual-Leone ${ }^{\mathrm{a}, \mathrm{c}}$ and Javier Cudeiro ${ }^{\mathrm{a}, \mathrm{b}, \mathrm{d}}$ \\ a Berenson-Allen Center for Noninvasive Brain Stimulation and Division of Cognitive Neurology, \\ Department of Neurology, Beth Israel Deaconess Medical Center, Harvard Medical School, Boston, MA, \\ USA \\ ${ }^{b}$ Neuroscience and Motor Control Group (NEUROcom), Institute for Biomedical Research (INIBIC), \\ Universidade da Coruña, A Coruña, Spain \\ ${ }^{c}$ Institut Guttman de Neurorehabilitació, Universitat Autónoma de Barcelona, Badalona, Barcelona, \\ SpaindCentro de Estimulación Cerebral de Galicia, A Coruña, Spain
}

\begin{abstract}
Transcranial static magnetic stimulation (tSMS) modulates cortical excitability probably by interacting with the GABA-glutamate intracortical balance. Different transcranial magnetic stimulation (TMS) waveforms probe distinct GABA-mediated cortical inhibition networks. The goal of the present work is to further characterize tSMS-induced changes in motor cortex reactivity and inhibition-excitation (I/E) balance. We hypothesized that tSMS affects particular cortical networks and thus, the effects of tSMS would be different depending on the TMS waveform used to assess its results. 23 healthy young adults completed two sessions of real or sham tSMS. The order of the sessions was randomized across participants. Motor evoked potentials (MEPs), cortical silent period (CSP), short- and long-interval intracortical inhibition (SICI and LICI), and intracortical facilitation (ICF) were assessed with TMS monophasic posterior-anterior (monoPA; $n=9$ ), monophasic anterior-posterior (monoAP; $n=7$ ), or biphasic (biAP-PA; $n=7$ ) pulses. Repeated measures analyses of variance and appropriate pairwise comparisons were performed for each TMS measure. After 15 min of real tSMS, the MEP amplitudes decreased compared to sham and baseline, SICI and LICI showed greater inhibition, and a tendency towards longer CSPs and less facilitation was found. These results were only observed with monoPA TMS. MEP amplitude increased compared to sham with monoAP TMS, with no clear changes in general intracortical I/E balance. Biphasic TMS was not able to capture any effects of tSMS The results show that the effects of tSMS on cortical excitability and inhibition involve specific interneuron circuits that are selectively activated by monoPA TMS. (C) 2019 IBRO. Published by Elsevier Ltd. All rights reserved
\end{abstract}

Key words:

transcranial static magnetic stimulation, transcranial magnetic stimulation, paired-pulse TMS, biphasic, monophasic, current direction. 
AP, Anterior-to-posterior; biAP-PA, Biphasic anterior-toposterior-posterior-to-anterior; CS, Conditioning stimulus; CSP, Cortical silent period; DMF, Dynamic magnetic field; EMG, Electromyography; FDI, First dorsal interosseous; FDR, False discovery rate; GABA, Gamma-Aminobutyric acid; HSD, Honestly significant difference; I/E, Inhibition-excitation balance; ICF, Intracortical facilitation; ISI, Inter-stimulus interval; LAI, Long afferent inhibition; LICI, Longinterval intracortical inhibition; MEP, Motor evoked potential; monoAP, Monophasic anterior-to-posterior; monoPA, Monophasic posterior-toanterior; PA, Posterior-to-anterior; rm-ANOVA, Repeated-measures analysis of variance; RMT, Resting motor threshold; SAI, Short afferent inhibition; SICF, Short-interval intracortical facilitation; SICI, Shortinterval intracortical inhibition; SMF, Static magnetic field; TMS, Transcranial magnetic stimulation; TS, Test stimulus; tSMS, Transcranial static magnetic stimulation.

\section{Introduction}

Magnetic fields can be classified into dynamic (DMF) and static (SMF), depending on whether there is a change of the direction or intensity of the field over time associated with an induced electric current. DMFs have been used for decades to explore brain function and physiology, as well as to modulate cortical activity. The most known example of this is transcranial magnetic stimulation (TMS).

During the last decade, several studies have found that moderate SMFs (i.e. magnetic fields between $1 \mathrm{mT}$ and $1 \mathrm{~T}$ (Rosen, 2003)) can modify human cortical excitability. The use of SMFs as a non-invasive brain stimulation tool has grown as a safe (Oliviero et al., 2015) and promising brain neuromodulation technique (Dileone et al., 2017, Lozano-Soto et al., 2018) named transcranial static magnetic stimulation (tSMS).

However, the way tSMS interacts with the cortical elements is insufficiently understood. The stimulation of motor cortex with a 0.5 -T magnet for 10-15 min induces $25 \%$ inhibition of motor evoked potentials (MEPs) (Oliviero et al., 2011). This reduction outlasts the intervention for several minutes and is negatively correlated with an increase in resting motor threshold (RMT) (Oliviero et al., 2011, Silbert et al., 2013), together translating in a decrease in motor cortex excitability. Nojima and colleagues (Nojima et al., 2015) investigated the effects of tSMS on short-interval intracortical inhibition (SICI) finding an enhancement of inhibition. Since SICI has been shown to be GABAA-dependent, the authors suggested that GABA-related interneurons may be responsible for the reduction of cortical excitability induced by tSMS. On the other hand, Arias and coworkers (Arias et al., 2017) found that other GABAA-dependent inhibitory TMS protocols (i.e. Short and long afferent inhibition, SAI and LAI, respectively) did not change after tSMS. The authors hypothesized that SICI and SAI or LAI protocols evaluate GABAA-inhibition but through independent interneuron cortical networks. More recently, Dileone et al. (Dileone et al., 2018) have shown that increasing the stimulation time to $30 \mathrm{~min}$ yield longer-lasting decrease in excitability but, in turn, less inhibition SICI and greater facilitation after short-interval intracortical facilitation (SICF). The authors proposed a more complex interaction between the SMFs and brain GABA-glutamate neurotransmitters balance.

TMS can be used to evaluate specific neural components and intracortical networks (Di Lazzaro et al., 2018) by choosing the appropriate waveform and current direction. The aim of the present study was to deepen on the understanding on tSMS-motor cortex interactions by using different TMS waveforms and current directions to evaluate tSMS-induced changes in cortical excitability and inhibition-excitation (I/E) balance. 


\section{Experimental Procedures}

\section{Participants}

Data were obtained from 26 healthy participants (ages 18 to 35, 12 males, 22 right-handed). All participants provided written informed consent prior to enrollment and received monetary compensation upon completion. The study was approved by the local Institutional Review Board and conducted in accordance with the Declaration of Helsinki.

Each participant completed two identical sessions (intersession interval 1-70 days; median $=10.5$ days) for sham and real tSMS. The order of the sessions was randomized and counterbalanced across participants. All participants underwent equivalent testing: (1) During the first visit, a structured medical history review and handedness determination were performed. Handedness was assessed with the revised Edinburgh Handedness Inventory (Oldfield, 1971). Safety screening for possible contraindications and side effects was performed at the beginning and end of each session. (2) After the initial evaluation, participants were assigned to one of three groups for TMS assessments, which differed only in the pulse waveform characteristics: ten subjects received monophasic posterior-anterior $\left(\right.$ mono $\left._{\mathrm{PA}}\right)$, nine monophasic anterior-posterior $\left(\mathrm{mono}_{\mathrm{AP}}\right)$, and seven biphasic $\left(\mathrm{bi}_{\mathrm{AP}-\mathrm{PA}}\right)$ stimulation (waveforms are named after the direction of the current induced in the motor cortex). TMS cortical reactivity and excitability assessments were acquired in the same order before and after real and sham tSMS intervention.

Three participants were excluded from the study and data analyses. One participant was excluded because of a recent episode of traumatic brain injury with probable loss of consciousness. The other two participants could not be included given their RMT was greater than $83 \%$ of the stimulator output and therefore stimulation at $120 \%$ RMT was not feasible. The remaining 23 participants had no history of neurological disease or any contraindication to TMS or tSMS

Participants were comfortably seated with their arms rested in a natural $90^{\circ}$ angle on a table in front of them. During the tSMS stimulation and TMS recordings, the participants were instructed to remain quiet with their muscles relaxed, monitored for drowsiness and asked to keep their eyes open.

\section{Transcranial static magnetic stimulation}

During each visit the participants were exposed to either real or sham tSMS. Each participant underwent both the real and sham interventions in a random order. The real tSMS was induced with a cylindrical neodymium magnet (Model DX8X8, K\&J Magnetics, US) (45 MGOe, nominal strength $65 \mathrm{~kg} \approx 0.5$ tesla-T). A non-magnetic metal replica indistinguishable from the real magnet was used for sham tSMS. Following prior publications (Oliviero et al., 2011, Silbert et al., 2013, Kirimoto et al., 2014) south polarity was used for stimulation. Both interventions had a duration of $15 \mathrm{~min}$ and were performed over the first dorsal interosseous (FDI) representation in primary motor cortex of the dominant hemisphere. The FDI cortical representation was previously identified by TMS motor output mapping (Rossini et al., 2015). Both the magnet and its replica were held in place with identical elastic bands under continuous investigators' monitoring. At the end of the second visit, participants were formally asked if they could determine which session was real and which sham. Only five out of the 23 analyzed participants (22\%) guessed correctly the order of real/sham visits. 


\section{EMG recordings}

Surface electromyography (EMG) activity was recorded from the dominant FDI using a PowerLab 4/25-T data acquisition device and Scope software (ADInstruments, Colorado Springs, CO, USA). The negative electrode was placed over the belly of the muscle, the positive electrode over the first interphalangeal joint of the second finger, and the ground over the ipsilateral ulnar styloid process.

EMG data were digitized at $1 \mathrm{kHz}$ for $250 \mathrm{~ms}$ following each stimulus trigger and amplified with a range of $\pm 10 \mathrm{mV}$ (band-pass filter $0.3-1000 \mathrm{~Hz}$ ). Triggered epochs were acquired for single and paired-pulse measures, while live EMG was recorded and monitored throughout the cortical silent period (CSP) protocol to provide feedback for muscle contraction. MEP peak-to-peak amplitudes ( $\mathrm{mV}$ ) for single- and paired-pulse protocols and CSP durations (ms) were measured for individual traces.

\section{Transcranial magnetic stimulation}

Neuronavigated-TMS was performed with a MagPro X100 (MagVenture A/S, Denmark) using a hand-held Cool-B65 figure-of-eight coil (outer diameter $75 \mathrm{~mm}$ ) placed over the motor cortex with the handle pointing backwards and at a $45^{\circ}$ angle. To assure consistent targeting throughout the experiment, we used a brain MRI template with a Brainsight TMS neuronavigation system (Rogue Research, Inc., QC, Canada) and a Polaris infrared-optical tracking system (Northern Digital Inc., ON, Canada).

Regardless of the TMS waveforms and current direction, the real and sham visits began with the assessment of the motor hotspot (optimal site for eliciting large and reliable motor responses on the FDI). The hotspot, once found, was marked in the template-MRI and designated thereafter as the neuronavigation target for the remaining of the visit. The hotspot was assessed also at the beginning of the second visit with the same methodology. RMT was determined in each session following hotspot assessment. RMT was defined following the International Federation of Clinical Neurophysiology guidelines (Rossi et al., 2009, Rossini et al., 2015) as the lowest intensity that elicits an MEP of at $\gg 50 \mu \mathrm{V}$ in at least $50 \%$ of the trials.

Once RMT was determined, a battery of standard TMS neurophysiological measures of cortical reactivity and excitability was acquired: baseline cortico-motor reactivity; contralateral CSP; and three common paired-pulse protocols interleaved in a pseudorandom sequence. After the 15-min tSMS or sham interventions these neurophysiological measures were repeated in the same order. For each TMS measure, individual data points $\gg 2.5 \mathrm{SD}$ from each participant's mean were excluded from calculation and analysis.

Baseline cortico-motor reactivity was assessed by the average peak-to-peak amplitude of 40 unconditioned pulses at $120 \%$ RMT. The 40 post-intervention unconditioned MEPs were expressed as a percentage of change from pre-intervention mean MEP amplitude and divided in four groups of 10 averaged consecutive trials. The change in cortico-motor reactivity will be hereafter referred to as MEP amplitude.

The CSP was assessed with 10 single pulses delivered at 120\%RMT during isometric contraction of the FDI at about $25 \%$ of the participant's total strength. Participants could rest for few seconds between pulses and had constant visual feedback of their performance with the live EMG. The CSP was measured from the onset of the MEP to the resumption of pre-TMS EMG activity (Orth and Rothwell, 2004), and the duration was averaged across all 10 trials. 
Paired-pulse protocols included SICI, long-interval intra-cortical inhibition (LICI) and intracortical facilitation (ICF) using standard parameters (Valls-Sole et al., 1992, Kujirai et al., 1993). SICI and ICF consisted of a conditioning stimulus (CS) at 80\%RMT, a test stimulus (TS) at $120 \%$ RMT and an interstimulus interval (ISI) of 3 and $12 \mathrm{~ms}$, respectively. In LICI, CS and TS were $120 \%$ RMT separated by an ISI of $100 \mathrm{~ms}$. Stimulation consisted of 40 individual trials per protocol (for a total of 120 trials), administered in a pseudorandom, interleaved order to reduce blocking effects and with pseudorandomized inter-trial interval (4-6s) to minimize expectation and avoid influence of a previous trial. The amplitude of the 40 conditioned MEP for each protocol was expressed as a percentage of unconditioned MEP amplitude and divided in four groups of 10 trials. The post-tSMS percentage of change from pre-intervention was then calculated. Post-to-pre paired-pulse change calculation will be referred to as SICI, LICI or ICF.

\section{Statistical analyses}

Stata software version 13.1 (StataCorp, College Station, TX, USA) was used for statistical analyses. Calculation of TMS data for each of the three waveforms/current directions (mono ${ }_{\mathrm{PA}}$, mono $_{\mathrm{AP}}$, bi $\mathrm{AP}_{\mathrm{APA}}$ ) included: \%change post- to pre-intervention of MEP amplitude; pre- and postintervention average CSP duration in ms; and \%change of paired-pulse measures (SICI, LICI, and ICF). All analyses were conducted using a two-tailed $95 \%$ confidence interval $(\alpha=0.05)$

All data were checked for normality using the Shapiro-Wilk test. MEP amplitude, LICI, SICI and ICF significantly deviated from normality $(p$ 's $<0.05)$, whereas CSP did not $(p$ 's $\gg 0.12)$. Consequently, MEP amplitude, LICI, SICI and ICF were transformed following previously described methods (van Albada and Robinson, 2007).

After data normalization, we conducted repeated-measures analyses of variance (rmANOVAs) to assess the effect of tSMS on cortical reactivity in both the real and sham visits. Each TMS measure was the dependent variable, the waveform/current direction, hereafter referred to as Waveform (mono PA , mono ${ }_{\mathrm{AP}}$, or bi $\mathrm{AP}_{\mathrm{APA}}$ ), was a between-subject variable with nested effects, and Intervention (real or sham tSMS) and Time (groups of 10 consecutive trials) were longitudinal within-subject variables. Follow-up Tukey's honestly significant difference (HSD) tests were used to conduct pairwise comparisons of the effects of the interventions for each different Waveform. Planned-contrast analyses were used to conduct pairwise comparisons of the intervention effects at each time point. Results were adjusted for multiple comparisons with the false discovery rate (FDR) method.

Finally, long periods of muscle relaxation (Todd et al., 2006) or possible cumulative effects of the single-pulse TMS (Pellicciari et al., 2015) have been shown to progressively increase MEP amplitude over time. We tested whether these factors have influenced our results by conducting an rm-ANOVA. MEP amplitudes after sham condition were included as the dependent variable, Waveform (mono $_{\mathrm{PA}}$, $\mathrm{mono}_{\mathrm{AP}}$, or $\mathrm{bi}_{\mathrm{AP}-\mathrm{PA}}$ ) as the between-subject variable with nested effects, and Time (groups of 10 consecutive trials) as the longitudinal within-subject variable.

\section{Results}

Table 1 shows the mean $( \pm$ SD) of each TMS measure before and after the intervention, and the effect and direction of the change due to the real/sham interventions. 
Table 1. Transcranial magnetic stimulation neurophysiological measures.

\begin{tabular}{|c|c|c|c|c|c|}
\hline & & & Pre-Intervention & Post-Intervention & Effect \\
\hline \multirow{6}{*}{ MEP amplitude } & \multirow{2}{*}{ monoPA } & Real & $1.4 \pm 0.9$ & $1.1 \pm 0.9$ & $\boldsymbol{\nabla} 24.4 \% * * *$ \\
\hline & & Sham & $1.2 \pm 0.6$ & $1.6 \pm 1.5$ & $\boldsymbol{\Delta} 41.3 \%$ \\
\hline & \multirow{2}{*}{ monoAP } & Real & $2.2 \pm 1.6$ & $2.5 \pm 1.3$ & А $14.7 \% *$ \\
\hline & & Sham & $2.1 \pm 1.3$ & $2.2 \pm 1.5$ & $\Delta 5 \%$ \\
\hline & \multirow{2}{*}{$B i A P-P A$} & Real & $1.8 \pm 0.5$ & $2.1 \pm 0.7$ & А $20 \%$ \\
\hline & & Sham & $1.5 \pm 0.9$ & $2.2 \pm 0.9$ & $\Delta 16.5 \%$ \\
\hline \multirow{6}{*}{$\mathrm{cSP}$} & \multirow{2}{*}{ monoPA } & Real & $119.4 \pm 32.5$ & $126.4 \pm 25.7$ & А $5.9 \% *$ \\
\hline & & Sham & $126.2 \pm 31.1$ & $136.4 \pm 27.6$ & $\boldsymbol{\Delta} 8.1 \% *$ \\
\hline & \multirow{2}{*}{ monoAP } & Real & $127.1 \pm 23.3$ & $143.5 \pm 50.8$ & $\Delta 12.9 \% *$ \\
\hline & & Sham & $133.4 \pm 36.8$ & $135.5 \pm 27.5$ & $\Delta 1.5 \% *$ \\
\hline & \multirow{2}{*}{$B i A P-P A$} & Real & $139.1 \pm 26.3$ & $147.1 \pm 18.3$ & $\Delta 5.8 \% *$ \\
\hline & & Sham & $138.7 \pm 28.1$ & $148.5 \pm 20.0$ & $\Delta 7.1 \% *$ \\
\hline \multirow{6}{*}{$\% \Delta \mathrm{LICI}$} & \multirow{2}{*}{ monoPA } & Real & $-0.8 \pm 0.1$ & $-0.9 \pm 0.1$ & А $3.3 \% * * *$ \\
\hline & & Sham & $-0.9 \pm 0.1$ & $-0.8 \pm 0.1$ & $\boldsymbol{\nabla} 0.8 \%$ \\
\hline & \multirow{2}{*}{ monoAP } & Real & $-0.8 \pm 0.4$ & $-0.8 \pm 0.4$ & А $1.6 \% * * *$ \\
\hline & & Sham & $-0.9 \pm 0.2$ & $-0.9 \pm 0.2$ & $\boldsymbol{\nabla} 0.6 \%$ \\
\hline & \multirow{2}{*}{$B i A P-P A$} & Real & $-0.9 \pm 0.2$ & $-0.9 \pm 0.1$ & $\Delta 6.5 \% * *$ \\
\hline & & Sham & $-0.9 \pm 0.2$ & $-0.9 \pm 0.2$ & А $1.5 \%$ \\
\hline \multirow{6}{*}{$\% \Delta \mathrm{SICI}$} & \multirow{2}{*}{ monoPA } & Real & $-0.7 \pm 0.3$ & $-0.7 \pm 0.2$ & ( $7.4 \% * * *$ \\
\hline & & Sham & $-0.7 \pm 0.2$ & $-0.6 \pm 0.2$ & $\boldsymbol{\nabla} 6.1 \%$ \\
\hline & \multirow{2}{*}{ monoAP } & Real & $-0.7 \pm 0.3$ & $-0.5 \pm 0.7$ & $\boldsymbol{\nabla} 31.8 \% * *$ \\
\hline & & Sham & $-0.6 \pm 0.4$ & $-0.6 \pm 0.5$ & $\boldsymbol{\nabla} 0.5 \%$ \\
\hline & \multirow{2}{*}{$B i A P-P A$} & Real & $-0.5 \pm 0.4$ & $-0.4 \pm 0.4$ & $\boldsymbol{\nabla} 22.3 \% * *$ \\
\hline & & Sham & $-0.6 \pm 0.3$ & $-0.4 \pm 0.6$ & $\boldsymbol{\nabla} 38.7 \%$ \\
\hline \multirow{6}{*}{$\% \Delta \mathrm{ICF}$} & \multirow{2}{*}{ monoPA } & Real & $0.04 \pm 0.4$ & $-0.1 \pm 0.5$ & $\boldsymbol{\nabla} 286.9 \%$ \\
\hline & & Sham & $0.2 \pm 0.4$ & $0.4 \pm 0.7$ & $\boldsymbol{\Delta} 145.3 \%$ \\
\hline & \multirow{2}{*}{ monoAP } & Real & $0.6 \pm 1.0$ & $1.1 \pm 1.9$ & $\boldsymbol{\Delta} 104.9 \%$ \\
\hline & & Sham & $0.3 \pm 0.6$ & $0.3 \pm 0.6$ & $\nabla 11.6 \%$ \\
\hline & \multirow{2}{*}{$B i A P-P A$} & Real & $0.8 \pm 0.7$ & $1.1 \pm 1.1$ & வ $29.4 \%$ \\
\hline & & Sham & $0.6 \pm 0.5$ & $1.1 \pm 0.8$ & А $87.6 \%$ \\
\hline
\end{tabular}

Mean and standard deviation (SD) are shown in the table. The black arrows indicate the \% of increase $(\boldsymbol{\Delta})$ or decrease $(\boldsymbol{\nabla})$ in mean MEP amplitude (mV) or cSP duration $(\mathrm{ms})$. For paired-pulse protocols the black arrows reflect the $\%$ of increase $(\boldsymbol{\Delta})$ or decrease $(\boldsymbol{\nabla})$ in mean inhibition (LICI, SICI) or facilitation (ICF). Abbreviations: \% $\Delta$, percentage of change from baseline; BiAP-PA, biphasic anterior-posterior-posterior-anterior; cSP, cortical silent period (ms); ICF, intracortical facilitation, MEP, motor evoked potential $(\mathrm{mV})$; monoAP, monophasic anterior-posterior; monoPA, monophasic posterior-anterior; LICI, long-interval intracortical inhibition; SICI, short-interval intracortical inhibition.

* Significant difference between pre and post measures at each intervention $(p<0.05)$.

** Significant difference between real and sham interventions $(\mathrm{p}<0.05)$. 
MEP amplitude inhibition after real tSMS was only observed when TMS was performed with mono $_{\text {PA }}$ (inhibition of $24.4 \%$, Table 1). The rm-ANOVA analysis for the MEP amplitude showed a significant effect of Waveform $(\mathrm{p}<0.001)$ and of Waveform-Intervention interaction $(\mathrm{p}<0.001)$. No significant effects of Intervention, Time or for the rest of the interactions were observed (all p's $\gg 0.05$ ). Post Hoc Tukey's HSD and planned contrast analyses, of Waveform and the Waveform-Intervention interaction respectively, showed a significant difference between mono $_{\mathrm{PA}}$ with both mono $\mathrm{AP}_{\mathrm{A}}$ and $\mathrm{bi}_{\mathrm{AP}-\mathrm{PA}}$ waveforms ( $\mathrm{p}$ 's $<0.05$ ). Mono $\mathrm{PA}_{\mathrm{PA}}$ was the only waveform that revealed significant inhibition after real intervention $(\mathrm{p}<0.001)$ whereas mono AP $_{\text {MEP amplitudes }}$ were significantly facilitated $(\mathrm{p}=0.02$, Fig. 1). Furthermore, the inhibitory effects of real tSMS when evaluated by mono ${ }_{\mathrm{PA}}$ were significantly greater than sham at Post-T3 and Post-T4 (i.e. pulses 21 to 30 and 31 to 40, respectively) (both p's $<0.02$, Fig. 1B). Follow-up contrast analysis for mono $_{\mathrm{AP}}$ waveform also showed an increase of mean MEP amplitude at real Post-T3 compared to sham condition although this change did not survive FDR correction $(p=0.18) . \mathrm{Bi}_{\mathrm{AP}}$ $\mathrm{PA}$ waveform did not differ significantly from $\mathrm{mono}_{\mathrm{AP}}$ and the slightly facilitatory effects of the tSMS captured by $\mathrm{Bi}_{\mathrm{AP}-\mathrm{PA}}$ did not reach significance.

A

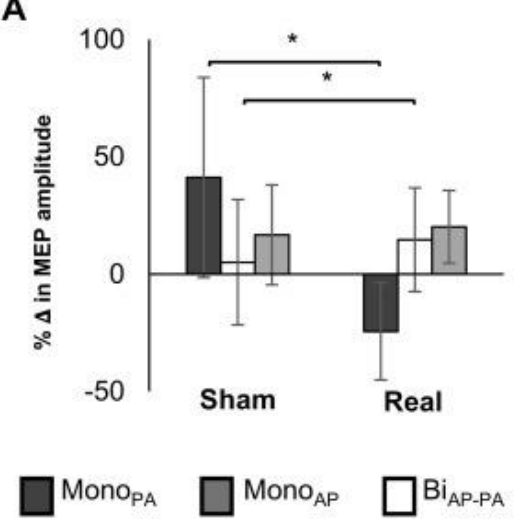

B

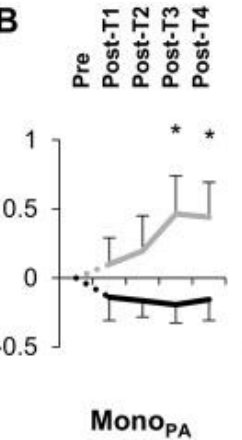

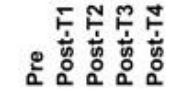

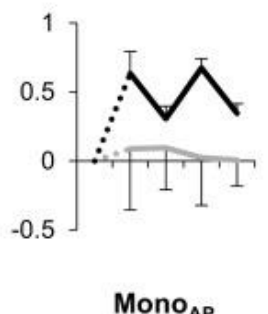

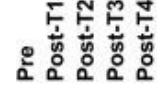

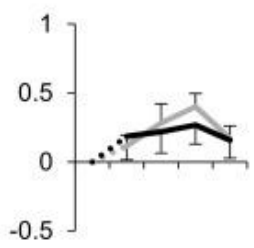

$\mathrm{Bi}_{\text {AP.PA }}$

Fig. 1. Effects of real tSMS and sham interventions on motor evoked potentials (MEP) amplitude. (A) Percentage of change $(\% \Delta)($ mean $\pm \mathrm{SE})$ from pre- to post-intervention evaluated in real and sham interventions for the three different waveforms. Negative values represent a decrease in MEP amplitude and an increase in inhibition in the motor cortex. (B) Comparison of the percentage of change from baseline $(\% \Delta)$ (mean $\pm \mathrm{SE}$ ) through time for the three different waveforms and current directions. Real intervention is shown in black and sham intervention is in light gray. The dashed lines represent the transition time from pre-intervention (Pre) to post-intervention (Post-T1-T4) where the intervention took place. Abbreviations: $\mathrm{Mono}_{\mathrm{AP}}$, monophasic anterior-posterior; $\mathrm{Bi}_{\mathrm{AP}-\mathrm{PA}}$, biphasic anterior-posterior-posterior-anterior; Mono $_{\mathrm{PA}}$, monophasic posterior-anterior.

When searching for possible influences of long periods of muscle relaxation or possible cumulative effects of TMS on sham MEP amplitude no significant differences were found for Waveform, Time or their interaction (all p's $\gg 0.3$ ).

All the Waveforms lengthened the duration of CSP regardless of the Intervention (Table 1). Accordingly, the rm-ANOVA for CSP showed a significant effect of Waveform $(\mathrm{p}<0.01)$ and Time $(\mathrm{p}=0.02)$ but no significant differences were found either for the Intervention or any of the interactions. Tukey's HSD for Waveform found that $\mathrm{Bi}_{\mathrm{AP}-\mathrm{PA}}$ was significantly different than both monophasic waveforms $(\mathrm{p}<0.05)$ which inhibited more. None of the rest of follow-up analyses were significant. 
All waveforms were able to elicit greater inhibition after the real intervention (Table 1) when performing LICI. Furthermore, both Waveform and Waveform-Intervention interaction were significant ( $\mathrm{p}$ 's $<0.001)$. Post hoc Tukey's HSD showed that all waveforms were significantly different form each other ( $\mathrm{p}$ 's $<0.05)$ but only the monophasic $\left(\right.$ mono $_{\mathrm{PA}}$ and $\left.\mathrm{mono}_{\mathrm{AP}}\right)$ were able to significantly inhibit after real tSMS (p's $<0.01$, Fig. 2A-B). The effects of Time and its interactions did not reach significance.

A

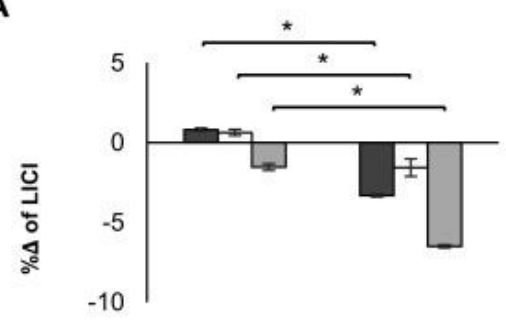

C

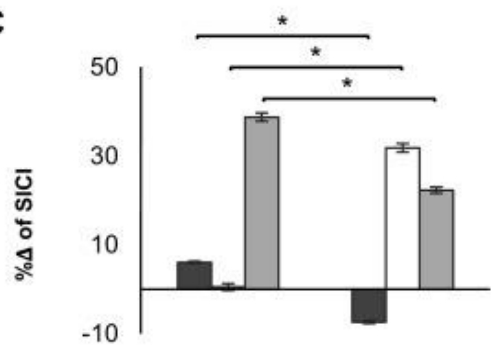

E

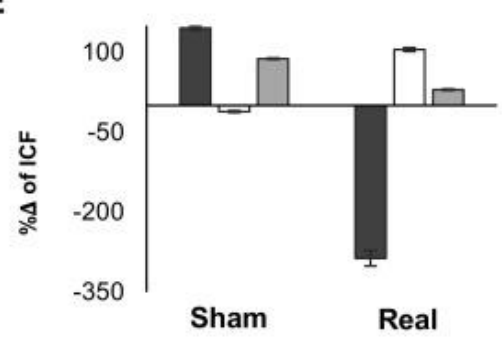

Mono $_{\mathrm{PA}} \square$ Mono $_{\mathrm{AP}} \quad \square \mathrm{Bi}_{\mathrm{AP}-\mathrm{PA}}$
B

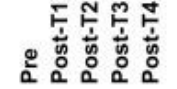

影影最
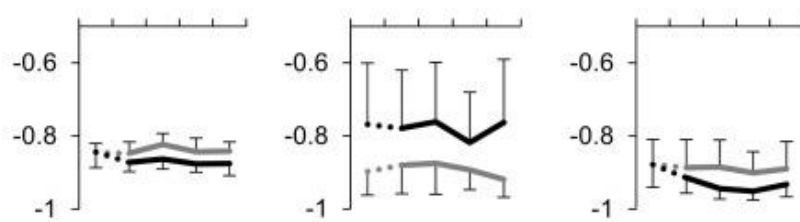

D
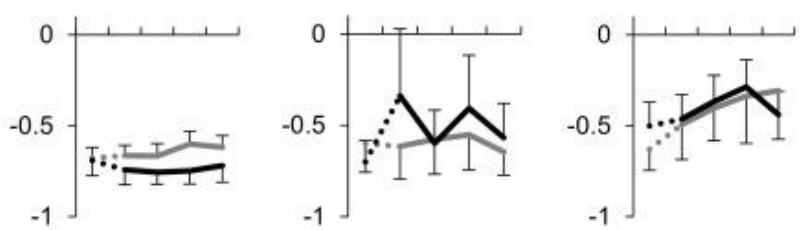

$\mathbf{F}$
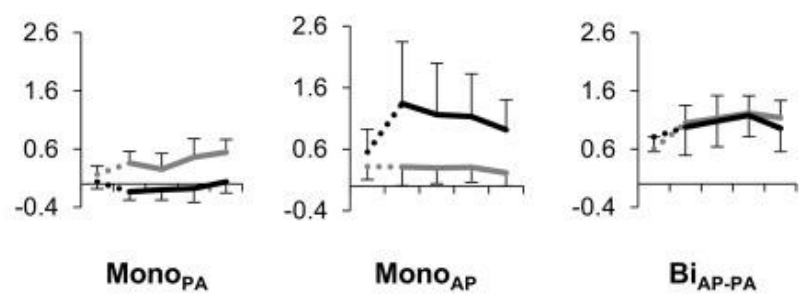

Real - Sham

Fig. 2. Effects of real tSMS and sham interventions on paired pulse TMS protocols. (A, C-D) Percentage of change (\% $\Delta)$ ) $($ mean $\pm \mathrm{SE})$ from pre- to post-intervention evaluated in real and sham interventions for the three different waveforms Negative values represent a decrease in motor evoked potentials (MEP) amplitude, in other words more inhibition after short- or long-interval intracortical inhibition (SICI or LICI, respectively) and less facilitation after intracortical facilitation (ICF). (B, D-F) Comparison of the percentage of change from baseline $(\% \Delta)$ (mean \pm SE) through time for the three different waveforms and current directions. Real intervention is shown in black and sham intervention is in light gray. The dashed lines represent the transition time from pre-intervention (Pre) to post-intervention (Post-T1-T4) where the intervention took place. Abbreviations: $\mathrm{Mono}_{\mathrm{AP}}$, monophasic anterior-posterior; $\mathrm{Bi}_{\mathrm{AP}-\mathrm{PA}}$, biphasic anterior-posteriorposterior-anterior; Mono ${ }_{\mathrm{PA}}$, monophasic posterior-anterior. 
When performing SICI, only mono ${ }_{\mathrm{PA}}$ waveform captured an increase in inhibition after real tSMS (Table 1). The rm-ANOVA yielded significant Waveform and WaveformIntervention interaction effects (both p's $<0.001$ ). Post Hoc Tukey's HSD analyses showed that $\mathrm{Bi}_{\mathrm{AP}-\mathrm{PA}}$ significantly differed from the other two waveforms $(\mathrm{p}<0.05)$. Planned contrast tests revealed a significant increase in inhibition after real tSMS for mono ${ }_{\mathrm{PA}}$ when compared to sham $(\mathrm{p}=0.005) . \mathrm{Bi}_{\mathrm{AP}-\mathrm{PA}}$ and mono $_{\mathrm{AP}}$, both showed a decrease in inhibition after both real and sham interventions. Nevertheless $\mathrm{Bi}_{\mathrm{AP}-\mathrm{PA}}$ led to a relative increase in inhibition after real when compared to sham tSMS $(p=0.005)$, whereas mono $_{A P}$ showed a significant decrease in inhibition (relative facilitation) after real intervention $(\mathrm{p}<0.001$, Fig. $2 \mathrm{C})$. None of the specific time points postintervention survived FDR correction (Fig. 2D).

Finally, among the TMS measures that were carried out, only ICF reflects the facilitatory mechanisms that take place in the motor system. Mono PA $_{\text {a }}$ was the only waveform that was able to capture an expected overall decrease in facilitation after real tSMS, while mono $_{\mathrm{AP}}$ and $\mathrm{bi}_{\mathrm{AP}}$ ${ }_{\mathrm{PA}}$ revealed some degree of facilitation (Table 1 and Fig. 2E-F). However, due to the high variance of the sample the rm-ANOVA yielded no significant effects of Waveform, Intervention, Time or their interactions ( $p$ 's $\gg 0.15$, Fig. 2E-F).

\section{Discussion}

The present study investigated the effects of real and sham tSMS on motor cortex excitability, expressed in terms of changes in MEP amplitude, and cortical I/E balance characterized by CSP, LICI, SICI and ICF. Different TMS waveforms and orientations were used to selectively explore distinct intracortical neural components and networks.

After the real intervention, the MEP amplitude and the paired-pulse protocols LICI and SICI showed an increase in intracortical inhibition, while CSP tended to have longer durations and ICF tended towards less facilitation of the conditioned MEPs. These effects of tSMS on the motor cortex excitability and inhibitory processes, however, were only revealed when explored with the

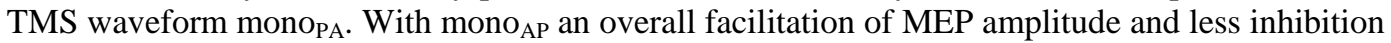
after SICI were observed; however, LICI induced more inhibition and no changes were observed in CSP or ICF. All together mono ${ }_{\mathrm{AP}} \mathrm{TMS}$ showed an increase in cortical excitability measured by single-pulse MEPs but no clear effect on I/E balance was observed. Finally, no significant tSMSinduced changes in excitability or I/E balance were captured by the bi $\mathrm{AP}_{\mathrm{AP}-\mathrm{PA}} \mathrm{TMS}$ waveform.

In order to understand the present findings, the best framework available nowadays is the theoretical canonical cortical model proposed by several authors in the past decades (Di Lazzaro et al., 2001a, Di Lazzaro et al., 2001b, Di Lazzaro et al., 2006, Di Lazzaro et al., 2008, Di Lazzaro et al., 2011, Di Lazzaro and Ziemann, 2013, Di Lazzaro et al., 2018). Although still uncertain, this cortical model suggests that depending on the TMS waveform and the current direction unique intracortical circuits are activated. As a brief example, mono ${ }_{\mathrm{PA}}$ and mono $_{\mathrm{AP}}$ activate different sets of interneurons that arrive at the PTN and motor pathway several milliseconds apart. From studies recording changes in spinal cord activity after a TMS pulse (Di Lazzaro and Rothwell, 2014, Di Lazzaro et al., 2018), it has been proposed that mono PA $_{\mathrm{PA}}$ activates the local intracortical interneurons in layers II and III eliciting primarily early I-waves, and particularly the I1-wave.

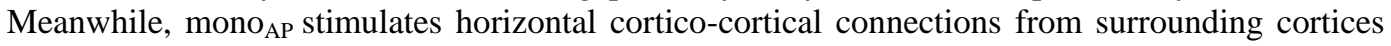
or from other brain structures to the primary motor cortex (such as premotor areas or the thalamus) eliciting mainly later I-waves with slightly delayed latencies (namely I2-I5 waves). By comparison, biphasic pulses evoke a more complex group of D- and I-waves where the intensity of the pulse plays a greater role and the combination of waves evoked by the AP and the PA components may have competing effects on the outcome. 
Previous studies on the effects of tSMS on the motor cortex excitability have found a decrease in MEP amplitude of about $25 \%$ after real tSMS. These results were first shown by Oliviero et al. (2011) and subsequently replicated by Silbert et al. (2013). Both groups used monoPA for their TMS procedures. Our results, in line with these prior reports, showed that the effects of the real tSMS yielded an average MEP amplitude decrease of $24.4 \%$ when the excitability of motor cortex was explored using the same type of waveform. In addition, we also explored the time course of these inhibitory effects and in relation to the responses to sham intervention. Relatively to sham, the inhibitory effects after the real tSMS were enlarged, showing a decrease up to a $60 \%$ when real and sham were directly compared. This difference between real and sham cortical excitability was not only due to a decrease of MEP amplitude after real tSMS, but also because there was a tendency towards MEP facilitation after sham. In contrast to the reduction of the MEP amplitude, the increase of MEPs following sham tSMS was captured by all waveforms and current directions as time went on with no significant difference between them. An increase of the motor responses after sham or no intervention has been previously related to prolonged periods of muscle relaxation (Todd et al., 2006) or possible cumulative effects of single-pulse TMS (Pellicciari et al., 2015). Todd et al. found an increase of about $50 \%$ of MEP average duration after 20 min of muscle relaxation with no increase in peripheral muscle response ( $\mathrm{M}$ wave) or spinal cord excitability ( $\mathrm{F}$ wave). The authors, who used monoPA TMS in their experiments, argued that this change could be mainly explained by an increase in cortical excitability due to the lack of motor input signals. Their hypothesis is also supported by previous experiments with ischemic or anesthetic nerve blocks (Brasil-Neto et al., 1992, Ziemann et al., 1998) where cortical excitability raised after a short period of time. In another relevant study, Pellicciari et al. (2015) studied the effects of blocks of single-pulses over time with fixed and random ISIs using biphasic TMS waveform. The authors found that regardless of the pattern of ITIs the MEP amplitude increased over time and argued that single-pulse TMS may have a modulatory cumulative effect on corticospinal excitability similar to the modulation seen after rTMS.

All of the above considerations suggest that the local SMFs of real tSMS are able not only to decrease cortical reactivity to some extent but also to prevent a rise in motor cortex excitability in response to either muscle inactivity or the cumulative facilitatory effects of repeated single-pulse TMS. In our results, this difference between real and sham for monoPA waveform was more evident at times T3 and T4 (about 4-6 min after the intervention). This time frame is well aligned with previous publications (Oliviero et al., 2011, Silbert et al., 2013).

While our results using monoPA TMS are in line with those previously reported, it is important to highlight that this decrease in motor cortex excitability was not observed with either monoAP or biAP-PA waveforms. Remarkably, the effects captured by monoAP were facilitatory showing an average increase of MEP amplitude after real tSMS. To explain this increase it is necessary to return to the canonical cortical model and the specific neural circuits that monoAP explores. As mentioned above, monoAP evaluates cortico-cortical connections that most probably have regulatory inputs from other cortical regions or brain structures. One possibility may be that the SMFs inhibit those regulatory inputs releasing cortico-cortical connections that are explored with monoAP, thus resulting in an overall MEP facilitation. This hypothesis should be further tested with future experiments, for example recording from spinal cord epidural electrodes to test whether there is an increase in the late I-waves amplitude after real tSMS when evaluated by monoAP. In contrast to monophasic TMS, MEP amplitudes assessed by biphasic TMS did not show any significant decrease or increase after the real tSMS or when compared to sham. In this case, a plausible explanation may be that biphasic pulses engage competing mechanisms by activating both the cortico-cortical connections (AP-component) and the inhibitory intracortical networks in layers II and III (PA-component).

Beyond cortical excitability, the balance between inhibition and excitation (I/E balance) within the motor system was evaluated. Single- and paired-pulse inhibitory protocols included SICI, LICI and CSP. These protocols have been associated with cortical GABA processes but the differences between them should be taken into consideration. GABA, which is the primary inhibitory neurotransmitter of the CNS, has two main membrane receptors: $\mathrm{GABA}_{\mathrm{A}}$ and $\mathrm{GABA}_{\mathrm{B}}$. While 
SICI is mediated by $\mathrm{GABA}_{\mathrm{A}}$ processes that exert a fast phasic ionotropic inhibition (Cherubini, 2010), LICI and CSP are mediated by $\mathrm{GABA}_{\mathrm{B}}$ translating slow metabotropic inhibitory processes (Mott, 2015). Furthermore, spinal cord epidural recordings have shown that SICI and LICI produce a reduction in the amplitude of late I-waves with preservation of the I1-wave. In the CSP protocol there is a first facilitatory phase where both the I1-wave and the late I-waves are enhanced with a posterior reduction or inhibitory phase (Di Lazzaro et al., 2018). On the other hand, facilitatory processes were evaluated by ICF. It has been proposed that the NMDA system is the main mediator in the facilitation produced by ICF (Ziemann et al., 2015). However, no particular changes have been observed in D- or I-waves on epidural recording studies (Di Lazzaro et al., 2018). To sum up, each inhibitory protocol despite their similarities most probably activates distinctive neural circuits through a common inhibitory neurotransmission system with specific membrane receptors, whereas facilitatory processes remain much less understood.

In a previous study, Nojima et al. (2015) evaluated the effects of tSMS on SICI, CSP and ICF when explored with a mono ${ }_{\mathrm{PA}}$ TMS waveform. Comparable to our mono ${ }_{\mathrm{PA}}$ results, the authors found greater inhibitory effects of SICI and a tendency towards longer CSP durations, suggesting that GABA-mediated inhibitory processes may be involved in the reduction of cortical excitability observed after real tSMS. However, a more recent study (Dileone et al., 2018) has shown that the effects of SMFs on SICI may be turned around by extending the time of stimulation implying that this relationship may be more complex than proposed before. The authors of the study suggested that tSMS may reduce glutamatergic excitation with short stimulation periods, whereas longer stimulations $(30 \mathrm{~min})$ may also reduce the phasic GABA-ergic inhibition. How these longer stimulation periods affect $\mathrm{GABA}_{\mathrm{B}}$-metabotropic processes (e.g. processes measured by LICI and CSP) still needs to be elucidated. CSP is a complex motor response that involves both cortical and spinal circuits with a facilitatory response followed by an inhibitory phase. Therefore, changes in CSP durations may be more difficult to unveil. Another complex response that involves cortical and spinal processes is ICF. However, the authors did not find any reduction in facilitation, whereas our results suggest a trend towards lower facilitation after real tSMS reflecting an overall cortical inhibition. It is probable that decreasing the variance of the sample (by either increasing the number of pulses, the $\mathrm{n}$ of the sample or modifying the technical parameters for a more stable ICF response) this reduction in facilitation may be more obvious. In regard to this last point, it has been suggested that a minimum of 30 pulses is required for a reliable response to ICF protocol (Biabani et al., 2018). Thus, in future studies a greater number of pulses should be used in order to further clarify whether facilitatory processes may be also affected by tSMS.

While the sample sizes may seem small, the power to detect significant differences has not been reduced. It should be noted that we were able to detect significant differences between the waveforms, and between real and sham interventions within each waveform. Also our samples are similar to those in previous studies. Furthermore, our results are in line and have expanded the results previously reported in the literature. A within-subject comparison of these parameters would have resulted in more power to detect significant differences in the effects of tSMS associated with pulse waveform and current direction. However, completing those studies using a within-subject design would have required six visits per subject, which may have reduced the feasibility of our study due to attrition.

In conclusion, tSMS reduces motor cortical excitability and modulates the intracortical inhibition/excitation balance towards inhibition, but these effects are only captured by TMS mono $_{\text {PA. }}$. These results suggest that tSMS affects processes linked to specific intracortical neural circuits. In other words, the effects of SMFs selectively affect specific networks of intracortical

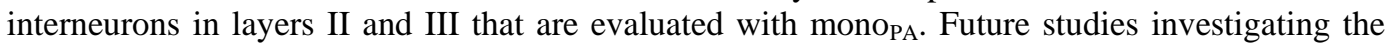
effects of tSMS on other muscle cortical representations, of longer stimulation periods on other paired-pulse protocols such as LICI or the effects of tSMS on epidural responses when TMS is performed with different waveforms and current directions might provide further insights on the effects of SMFs on specific cortical circuits. 


\section{Acknowledgments}

We thank Margo Bernstein (BIDMC) and Krista Grobelny (BIDMC) for assistance with data collection, and Ann Connor (BIDMC) for regulatory oversight and compliance, and assistance with evaluation of participants' health and medical history.

\section{Author Contributions}

P.D-P, A.P-L and J.C. contributed to study concept and design. P.D-P contributed to data collection. P.D-P, A.P-L and J.C. contributed to the data analysis. P.D-P, A.P-L and J.C. contributed to interpretation of the data. P.D-P drafted the manuscript. P.D-P, A.P-L and J.C. contributed to revising the manuscript.

\section{Funding}

J.C. was supported in part by a Salvador de Madariaga Fellowship for visiting scientists (Ministry of Education, Culture and Sport, Spain). A.P.-L. was supported in part by the Sidney R. Baer Jr. Foundation, the National Institutes of Health (NIH R01 MH100186, R01 NS073601, R01 HD069776, R21 MH099196, R21 NS082870; R21 NS085491, R21 HD07616), and Harvard Catalyst | The Harvard Clinical and Translational Science Center (NCRR and the NCATS NIH, UL1 RR025758). The content is solely the responsibility of the authors and does not necessarily represent the official views of Harvard Catalyst, Harvard University and its affiliated academic health care centers, or any of the listed granting agencies.

\section{Conflict of interest}

A.P.-L. serves on the scientific advisory boards for Neuronix, Starlab Neuroscience, Neuroelectrics, Constant Therapy, Cognito, NovaVision, and Neosync; and is listed as inventor on several issued and pending patents on real-time integration of transcranial magnetic stimulation with electroencephalography and magnetic resonance imaging. The remaining authors declare no competing interests. 


\section{References}

1. P Arias, L Adan-Arcay, B Puerta-Catoira, A Madrid, J CudeiroTranscranial static magnetic field stimulation of M1 reduces corticospinal excitability without distorting sensorimotor integration in humans. Brain Stimulat, 10 (2017), pp. 340-342

2. M Biabani, M Farrell, M Zoghi, G Egan, S Jaberzadeh. The minimal number of TMS trials required for the reliable assessment of corticospinal excitability, short interval intracortical inhibition, and intracortical facilitation. Neurosci Lett, 674 (2018), pp. 94-100

3. JP Brasil-Neto, LG Cohen, A Pascual-Leone, FK Jabir, RT Wall, M Hallett. Rapid reversible modulation of human motor outputs after transient deafferentation of the forearm: a study with transcranial magnetic stimulation. Neurology, 42 (1992), pp. 1302-1306

4. E Cherubini. Phasic GABAA-mediated inhibition. Epilepsia, 51 (2010), p. 13

5. V Di Lazzaro, JC Rothwell. Corticospinal activity evoked and modulated by non-invasive stimulation of the intact human motor cortex. J Physiol, 592 (2014), pp. 4115-4128

6. V Di Lazzaro, U Ziemann. The contribution of transcranial magnetic stimulation in the functional evaluation of microcircuits in human motor cortex. Front Neural Circuits, 7 (2013), p. 18

7. V Di

Lazzaro, A Oliviero, P Mazzone, A Insola, F Pilato, E Saturno, A Accurso, P Tonali, J Rothw ell. Comparison of descending volleys evoked by monophasic and biphasic magnetic stimulation of the motor cortex in conscious humans. Exp Brain Res, 141 (2001), pp. 121-127

8. V Di

Lazzaro, A Oliviero, E Saturno, F Pilato, A Insola, P Mazzone, P Profice, P Tonali, J Rothwel 1. The effect on corticospinal volleys of reversing the direction of current induced in the motor cortex by transcranial magnetic stimulation. Exp Brain Res, 138 (2001), pp. 268-273

9. V Di Lazzaro, F Pilato, A Oliviero, M Dileone, E Saturno, P Mazzone, A Insola, P Profice, F Ranieri, F Capone, PA Tonali, JC Rothwell. Origin of facilitation of motor-evoked potentials after paired magnetic stimulation: direct recording of epidural activity in conscious humans. $\mathrm{J}$ Neurophysiol, 96 (2006), pp. 1765-1771

10. V Di Lazzaro, U Ziemann, RN Lemon. State of the art: physiology of transcranial motor cortex stimulation. Brain Stimulat, 1 (2008), pp. 345-362

11. V Di Lazzaro, P Profice, F Ranieri, F Capone, M Dileone, A Oliviero, F Pilato. I-wave origin and modulation. Brain Stimulat, 5 (2011)

12. V. Di Lazzaro, J. Rothwell, M. Capogna. Noninvasive stimulation of the human brain: activation of multiple cortical circuits. Neuroscientist, 24 (3) (2018), pp. 246-260

13 M Dileone, MC Carrasco-Lopez, JC Segundo-Rodriguez, L Mordillo-Mateos, N LopezAriztegui, F Alonso-Frech, MJ Catalan-Alonso, JA Obeso, A Oliviero, G Foffani. Dopaminedependent changes of cortical excitability induced by transcranial static magnetic field stimulation in Parkinson's disease. $\quad$ Sci Rep, 7 (2017), p. 4329

14. M Dileone, L Mordillo-Mateos, A Oliviero, G Foffani. Long-lasting effects of transcranial static magnetic field stimulation on motor cortex excitability. Brain Stimulat, 11 (2018), pp. 676-688

15. H Kirimoto, H Tamaki, T Matsumoto, K Sugawara, M Suzuki, M Oyama, H Onishi. Effect of transcranial static magnetic field stimulation over the sensorimotor cortex on somatosensory evoked potentials in humans. Brain Stimulat, 7 (2014), pp. 836-840

16. T Kujirai, MD Caramia, JC Rothwell, BL Day, PD Thompson, A Ferbert, S Wroe, P Asselman, CD Marsden. Corticocortical inhibition in human motor cortex. J Physiol, 471 (1993), pp. 501-519

17. E. Lozano-Soto, V. Soto-León, S. Sabbarese, L. Ruiz-Alvarez, M. Sanchez-del-Rio, J. Aguilar, Strange B.A., Foffani G., A. Oliviero. Transcranial static magnetic field stimulation (tSMS) of the visual cortex decreases experimental photophobia. Cephalalgia, 38 (8) (2018), pp. 1493-1497

18. D Mott. The metabotropic GABAB receptors. (2015)

19. I Nojima, S Koganemaru, H Fukuyama, T Mima. Static magnetic field can transiently alter the human intracortical inhibitory system. Clin Neurophysiol, 126 (12) (2015), pp. 2314-2319

20. RC Oldfield. The assessment and analysis of handedness: the Edinburgh inventory. Neuropsychologia, 9 (1971), pp. 97-113

21. A Oliviero, L Mordillo-Mateos, P Arias, I Panyavin, G Foffani, J Aguilar. Transcranial static magnetic field stimulation of the human motor cortex. J Physiol, 589 (2011), pp. 4949-4958 
22. A Oliviero, MC Carrasco-López, M Campolo, YA Perez-Borrego, V Soto-León, JJ GonzalezRosa, AM Higuero, BA Strange, J Abad-Rodriguez, G Foffani. Safety study of transcranial static magnetic field stimulation (tSMS) of the human cortex. Brain Stimulat, 8 (2015), Article 481485

23. M Orth, J Rothwell. The cortical silent period: intrinsic variability and relation to the waveform of the transcranial magnetic stimulation pulse. Clin Neurophysiol, 115 (2004), pp. 1076-1082

24. M Pellicciari, C Miniussi, C Ferrari, G Koch, M Bortoletto. Ongoing cumulative effects of single TMS pulses on corticospinal excitability: an intra- and inter-block investigation. Clin Neurophysiol Off J Int Fed Clin Neurophysiol, 127 (2015), pp. 621-628

25. AD Rosen. Mechanism of action of moderate-intensity static magnetic fields on biological systems. Cell Biochem Biophys, 39 (2003), pp. 163-173

26. S Rossi, M Hallett, PM Rossini, A Pascual-Leone. Safety, ethical considerations, and application guidelines for the use of transcranial magnetic stimulation in clinical practice and research.

Clin Neurophysiol Off J Int Fed Clin Neurophysiol, 120 (2009), pp. 2008-2039

27. PM Rossini, et al. Non-invasive electrical and magnetic stimulation of the brain, spinal cord, roots and peripheral nerves: basic principles and procedures for routine clinical and research application. An updated report from an I.F.C.N. committee. Clin Neurophysiol Off J Int Fed Clin Neurophysiol, 126 (2015), pp. 1071-1107

28. Silbert BI, Pevcic DD, Patterson HI, Windnagel KA, Thickbroom GW (2013). Inverse correlation between resting motor threshold and corticomotor excitability after static magnetic stimulation of human motor cortex. Brain Stimulat. Available at: https://doiorg.accedys.udc.es/10.1016/j.brs.2013.03.007.

29. G Todd, JE Butler, SC Gandevia, JL Taylor. Decreased input to the motor cortex increases motor cortical excitability. Clin Neurophysiol Off J Int Fed Clin Neurophysiol, 117 (2006), pp. 2496-2503

30. J Valls-Sole, A Pascual-Leone, EM Wassermann, M Hallett. Human motor evoked responses to paired transcranial magnetic stimuli. Electroencephalogr Clin Neurophysiol, 85 (1992), pp. 355-364

31. SJ van Albada, PA Robinson. Transformation of arbitrary distributions to the normal distribution with application to EEG test-retest reliability. J Neurosci Methods, 161 (2007), pp. 205-211

32. U Ziemann, B Corwell, LG Cohen. Modulation of plasticity in human motor cortex after forearm ischemic nerve block. J Neurosci Off J Soc Neurosci, 18 (1998), pp. 1115-1123

33. U Ziemann, J Reis, P Schwenkreis, M Rosanova, A Strafella, R Badawy, F Muller-Dahlhaus. TMS and drugs revisited 2014. Clin Neurophysiol Off J Int Fed Clin Neurophysiol, 126 (2015), pp. 1847-1868

\section{Glossary}

Cortical silent period (CSP)

period of electrical silence in electromyogram that follows the activation of the corticospinal tract after a cortical stimulus during tonic contraction of the target muscle.

D-wave

evoked potential recorded at cervical spinal level, evoked by a stimulus over motor cortex (e.g. a transcranial magnetic stimulation pulse), which represents direct activation of the pyramidal tract neuron.

\section{I-waves}

evoked potentials recorded at cervical spinal level, evoked by a stimulus over the motor cortex (e.g. a transcranial magnetic stimulation pulse), which represent probable activation of cortical interneurons leading to the indirect activation of the pyramidal tract neuron.

Intracortical facilitation (ICF)

enhancement of the motor evoked potential following a pair of transcranial magnetic stimulation pulses over the motor cortex when the first stimulus has a low intensity and the inter-stimulus interval is between 8 and $30 \mathrm{~ms}$. 
Long-interval intracortical inhibition (LICI)

suppression of the motor evoked potential following a pair of transcranial magnetic stimulation pulses over the motor cortex when both stimuli have sufficient intensity and with an interstimulus interval between 50 and $200 \mathrm{~ms}$.

Resting motor threshold (RMT)

the minimum intensity at which there is a motor response after at least half of the stimuli.

Short-interval intracortical inhibition (SICI)

the suppression of the motor evoked potential following a pair of transcranial magnetic stimulation pulses over the motor cortex when the first stimulus has a low intensity and the interstimulus interval is between 1 and $4 \mathrm{~ms}$.

TMS pulse waveform

refers to the shape of the TMS pulse. Most commonly available waveforms are biphasic (the pulse is sinusoidal and has both positive and negative phases) or monophasic (the pulse is not sinusoidal and has a prominent positive or negative phase).

TMS pulse current direction

refers to the direction of the TMS-induced electrical current in relation to the scalp. Most commonly used current directions are posterior-to-anterior and anterior-to-posterio 